
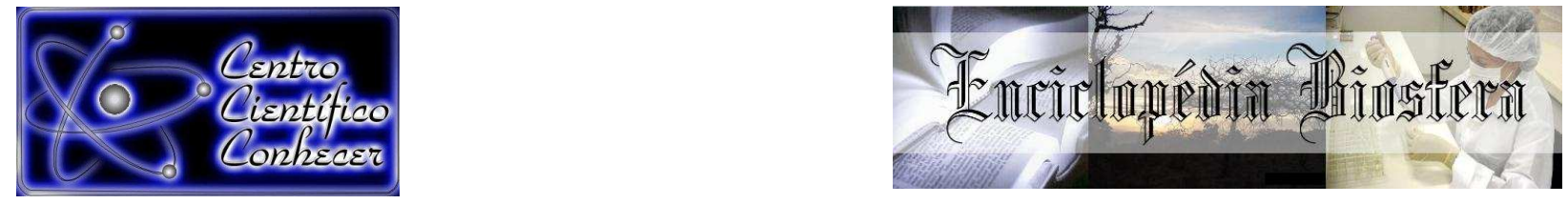

\title{
DESENVOLVIMENTO DE MUDAS DE MANDACARU SOB DIFERENTES NÍVEIS DE SALINIDADE E SOMBREAMENTO
}

\author{
Rosana Martins Portela ${ }^{1}$, Luciana Ferreira de Lima ${ }^{1}$, Jéssica Soares Pereira ${ }^{2}$, Natália \\ Almeida Lima ${ }^{3}$, Roberto Jun Takane ${ }^{4}$ \\ ${ }^{1}$ Engenheira Agrônoma - voluntária do Projeto CEFLOR - Universidade Federal do \\ Ceará, Fortaleza - Brasil (rosanatda@gmail.com) \\ ${ }^{2}$ Pós-Graduando em Agronomia - Fitotecnia \\ ${ }^{3}$ Graduando em Agronomia \\ ${ }^{4}$ Professor do Departamento de Fitotecnia
}

Recebido em: 08/09/2015 - Aprovado em: 14/11/2015 - Publicado em: 01/12/2015

DOI: http://dx.doi.org/10.18677/Enciclopedia_Biosfera_2015_131

\begin{abstract}
RESUMO
O mandacaru (Cereus jamacaru D. C.) vem ganhando destaque como planta ornamental predominante de regiões secas, onde a salinidade é um fator limitante da produção agrícola. A presente pesquisa objetivou avaliar o desenvolvimento de mudas de mandacaru sob diferentes níveis de salinidade e sombreamento. $O$ delineamento experimental foi o inteiramente casualizado (DIC), disposto em um arranjo fatorial $3 \times 5$ (tipos de telado e cinco níveis de condutividade elétrica), com quatro repetições de 4 plantas cada. Os dados foram submetidos à análise de variância e as médias comparadas pelo teste de Tukey ao nível de 5\% de probabilidade. Os tratamentos consistiram de diferentes níveis de salinidade em água corrente: $\mathrm{S}_{0}$ - controle $(0,0) ; \mathrm{S}_{1}\left(1-1,3 \mathrm{dS} \mathrm{m} \mathrm{m}^{-1}\right) ; \mathrm{S}_{2}\left(2-2,3 \mathrm{dS} \mathrm{m}^{-1}\right) ; \mathrm{S}_{3}(3-3,3 \mathrm{dS} \mathrm{m}$ $\left.{ }^{1}\right)$ e $S_{4}\left(4-4,4 \mathrm{dS} \mathrm{m} \mathrm{m}^{-1}\right)$ em três tipos de telado: 30,50 e $70 \%$. As avaliações de sobrevivência foram realizadas quinzenalmente. Diariamente foram mensuradas temperatura, UR\% do ar e luminância através dos instrumentos termo-higrômetro e luxímetro. As variáveis analisadas foram: número de plantas vivas e mortas (avaliação de sobrevivência), altura das plantas, diâmetro do caule, matéria fresca total, massa seca da parte aérea e da raiz. O sombreamento de $30 \%$ foi o que proporcionou menor sobrevivência das mudas de mandacaru, porém, nos de 50 e $70 \%$ houve maior sobrevivência das plântulas. A salinidade interferiu em todos os parâmetros analisados, podendo-se afirmar que essa espécie é sensível a baixas concentrações salinas sob condições de alta temperatura e umidade.
\end{abstract}

PALAVRAS-CHAVE: Condutividades; interações, temperatura. 


\title{
DEVELOPMENT OF SEEDLINGS MANDACARU UNDER DIFFERENT LEVELS OF SALINITY AND SHADE
}

\begin{abstract}
The mandacaru (Cereus jamacaru DC) is gaining prominence as the predominant ornamental plant dry areas where salinity is a limiting factor of agricultural production. This research aimed to evaluate the development of seedlings mandacaru under different levels of salinity and shading. The experimental design was completely randomized (DIC), followed by a $3 \times 5$ factorial arrangement ( 3 types of greenhouse $x$ 5 levels of electrical conductivity), with four replicates of 4 plants each. Data were subjected to analysis of variance and means compared by Tukey test at $5 \%$ probability. The treatments consisted of different salinity levels in tap water: S0 control (0.0); S1 (1-1.3 dS m-1); S2 (2-2.3 dS m-1); S3 (3 - $3.3 \mathrm{dS} \mathrm{m-1)} \mathrm{and} \mathrm{S4} \mathrm{(4} \mathrm{-}$ $4.4 \mathrm{dS} \mathrm{m}-1)$ in three types of greenhouse 30,50 and $70 \%$. Survival of evaluations were carried out fortnightly, from the first application. Daily were measured temperature, $\mathrm{RH} \%$ of air and luminance through the instruments thermo-hygrometer and light meter. The variables analyzed were: number of live and dead plants (evaluating survival), plant height, stem diameter, total fresh matter, dry matter of shoot and root. The shading was $30 \%$ which resulted in lower survival mandacaru seedlings, however, in 50 and $70 \%$ was most seedling survival. Salinity interfered in all parameters analyzed, can be said that this species is sensitive to low salt concentrations under high temperature and humidity conditions.
\end{abstract}

KEYWORDS: conductivities, interactions, temperature.

\section{INTRODUÇÃO}

O cultivo de plantas ornamentais no Brasil é praticado por pequenos e médios produtores utilizando-se cerca de 11.800 mil hectares por ano em mais de 400 municípios (IBRAFLOR, 2013). As cactáceas são bem apreciadas como plantas ornamentais e diversas espécies endêmicas do Brasil têm sido indicadas como potenciais para tal fim, atraindo vários colecionadores de todos os locais, devido a sua facilidade de cultivo, rusticidade e beleza (SOUZA \& LORENZI, 2005). O mesmo autor acrescenta afirmando que dentro da caatinga nordestina diversas espécies estão entre as mais típicas, dentre elas o mandacaru (Cereus jamacaru D.C.), com especialidade de sobreviver em ambientes secos. SANCHES et al., (2007) complementam quando afirmam que tais espécies vêm ganhando espaço na floricultura pela facilidade em todo o seu ciclo produtivo e por suportar as condições climáticas predominantes da região, ou seja, escassez de água e altas temperaturas.

O mandacaru é um cacto colunar, nativo da caatinga, extremamente rústico e geralmente encontrado em catingueiras arbóreas, onde quase não há presença de solos. Lembra um candelabro por conter um eixo principal pouco curvado com ramos assimétricos, o que garante formato em pertencer ao gênero Cereus, que significa círio/vela. Possui resistência a extensos períodos de seca, não o impedindo de crescer e frutificar. É pertencente à família das cactáceas formando a paisagem típica do semiárido nordestino (CAVALCANTI et al., 2008).

Quando se trata das condições naturais às quais essas espécies estão habituadas, vale ressaltar que as regiões áridas e semiáridas merecem destaque em todo o mundo, pois a salinidade nesses locais tem sido um fator limitador da produção agrícola. Isso porque a evapotranspiração por ser maior que a precipitação pluvial, contribui com um déficit hídrico por quase todo o ano, não lavando os sais 
que residem no perfil do solo (NOGUEIRA FILHO et al., 2012). Segundo VIÉGAS et al. (2001) esse fenômeno tem contribuído ainda mais para o aumento do problema da salinização de solos, juntamente com um manejo impróprio da água de irrigação, complementam NOGUEIRA FILHO et al. (2012). SHANNON et al. (1994) garantem que a planta responde a salinidade até este momento por influência de interações do ambiente como: temperatura, umidade relativa e radiação.

A luminosidade é um fator ambiental extremamente importante para o crescimento das plantas, que gera biomassa vegetal por converter energia luminosa em energia química, participa do processo de fotossíntese, abertura de estômatos e síntese de clorofila. Pode também causar estresse às mesmas se proporcionadas de forma irregular (NOGUEIRA FILHO et al., 2012; FREITAS et al., 2012). De acordo com ATROCH et al. (2001), níveis de luminosidade alterados podem colocar em risco a produtividade do vegetal através de interferências que podem ocorrer em respostas anormais de crescimento, anatômicas, fisiológicas e bioquímicas. De acordo com SEARTE et al. (2011); AASAMAA; SÕBER (2011) a maioria dos vegetais desenvolve capacidade para habituar-se às diferenciações de luminosidade e temperatura, a fim de regularizar abertura e fechamento estomático.

Diante da importância mandacaru (Cereus jamacaru) na cadeia de flores e plantas ornamentais considerando seu desenvolvimento em ambientes salinos, a presente pesquisa objetivou avaliar o desenvolvimento de mudas de mandacaru em condições de diferentes níveis de salinidade e sombreamento.

\section{MATERIAL E MÉTODOS}

O experimento foi desenvolvido na Universidade Federal do Ceará - UFC, Campus do Pici, Fortaleza - CE, localizada à longitude de 37\%48' W, latitude 336' S e altitude aproximadamente de $21 \mathrm{~m}$. Segundo KÖPPEN (1948), o clima é do tipo Aw', tropical chuvoso, com temperatura e precipitação média anual de $27^{\circ}$ e 1.338 $\mathrm{mm}$, respectivamente. O ensaio foi conduzido no período de dezembro de 2014 a março de 2015, em casa de vegetação pertencente à UFC. A casa de vegetação é coberta com plástico leitoso, difusor de 150 micras, com sombrites de 30, 50 e $70 \%$ de retenções de luminosidade, aproximadamente, acomodados em locais diferentes dentro da estrutura.

O delineamento experimental utilizado foi o Inteiramente Casualizado (DIC), seguindo-se de um arranjo fatorial $3 \times 5$ (3 tipos de telado $\times 5$ níveis de condutividade elétrica), com quatro repetições de 4 plantas cada, totalizando 15 tratamentos no geral, com 16 plantas para cada tratamento realizado.

A espécie de mandacaru utilizada no trabalho foi Cereus jamacaru D. C., cujas sementes foram coletadas na cidade de Santana de Acaraú em março de 2013, que foram semeadas em recipientes de plástico, em substrato pó de coco umedecido. Depois da semeadura os recipientes foram fechados em sacos plásticos de 50 micras e colocados à sombra, formando um tipo de câmara úmida para favorecer a embebição das sementes e as plântulas permanecerem em bom estado até o momento do transplantio.

No dia 26 de dezembro de 2014, as mudas com um ano e oito meses de idade foram transplantadas para bandejas de poliestireno com 200 células, comprimento/largura da célula: $31,0 \times 31,0 \mathrm{~mm}$ e volume $18 \mathrm{~cm}^{3}$, sendo utilizadas apenas 100 células de cada bandeja, a fim de organizar melhor e padronizar os tratamentos para as mudas. No total utilizaram-se três bandejas. Na instalação do experimento placas devidamente identificadas foram sorteadas em células alternadas, totalizando 25 tratamentos em cada bandeja. Após o transplantio, cada 
bandeja permaneceu em um tipo de telado presente na casa de vegetação $(30,50$ e $70 \%$, ou seja, mudas que permaneceram em telado de $30 \%$, receberam $70 \%$ de luminosidade; as que permaneceram em telado de $50 \%$ receberam $50 \%$ de luminosidade e as que permaneceram em telado de $70 \%$, receberam $30 \%$ de luminosidade. Os tratamentos consistiram de diferentes condutividades elétricas em água corrente: $\mathrm{S}_{0}$ - controle $(0,0) ; \mathrm{S}_{1}\left(1-1,3 \mathrm{dS} \mathrm{m}^{-1}\right) ; \mathrm{S}_{2}\left(2-2,3 \mathrm{dS} \mathrm{m}^{-1}\right) ; \mathrm{S}_{3}(3-3,3 \mathrm{dS}$ $\left.\mathrm{m}^{-1}\right)$ e $\mathrm{S}_{4}\left(4-4,4 \mathrm{dS} \mathrm{m}^{-1}\right)$ em três tipos de telado: 30,50 e $70 \%$.

As soluções salinas foram preparadas diluindo-se $\mathrm{NaCl}$ em água até a obtenção da condutividade elétrica, obtida através de um auxílio de um condutivímetro. As aplicações iniciaram quinze dias após o transplantio, adicionando-se $10 \mathrm{~mL}$ em cada célula da bandeja com auxílio de uma seringa. As avaliações de sobrevivência foram realizadas quinzenalmente, contando-se a partir da primeira aplicação. Diariamente foram mensuradas a temperatura e umidade relativa do ar no local do experimento, ou seja, na própria casa de vegetação, e a luminância/fontes de luz nos diferentes ambientes com o auxílio dos instrumentos termo-higrômetro e um luxímetro para se obter a média e determinar a que condições as mudas apresentaram melhores resultados na produção de sua biomassa verde.

As variáveis analisadas foram: número de plantas vivas e mortas (avaliação de sobrevivência): para essa análise considerou-se a contagem de plantas vivas e mortas de cada tratamento a partir do décimo quinto dia após a primeira aplicação da solução salina, sendo no total realizadas 5 avaliações de sobrevivência. As variáveis morfológicas analisadas foram as seguintes: altura das plantas (AP), realizada com o auxílio de uma régua graduada, sendo os dados expressos em $\mathrm{cm}^{2}$; diâmetro do caule (DC), feita com o auxílio de um paquímetro digital de precisão, sendo os dados expressos em mm; matéria fresca total (MFT), as plantas foram retiradas do substrato para em seguida serem pesadas em balança analítica $(0,001 \mathrm{~g})$; massa seca da parte aérea e da raiz (MSPA; MSR), as plantas foram colocadas para secar em estufa de circulação forçada a uma temperatura de $8^{\circ} \mathrm{C}$ por um período de 48 horas, até atingirem peso constante. Após isso as amostras foram pesadas em balança analítica $(0,001 \mathrm{~g})$.

Os dados foram submetidos à análise de variância utilizando o Software de análise estatística Sisvar®, versão 5.3 (FERREIRA, 2010). As comparações entre os três tipos de telado foram realizadas pelo teste de Tukey ao nível de $5 \%$ de probabilidade. Para a confecção dos gráficos foi utilizado o programa Microsoft Office Excel 2007.

\section{RESULTADOS E DISCUSSÃO}

Pode-se observar quanto aos caracteres morfoagronômicos de altura da planta (AP), diâmetro do caule (DC), matéria fresca total (MFT), massa seca da parte aérea (MSPA) e matéria seca da raiz (MSR), que para AP houve efeito significativo aos níveis de $5 \%$ e $1 \%$ de significância para os fatores doses (D) e telados (T), respectivamente. Para DC, MSPA e MSR as doses não apresentaram significância (TABELA 1). 
TABELA 1 - Resumo das análises de variância de altura de planta (AP), diâmetro do caule (DC), massa fresca total (MFT), massa seca da parte aérea (MSPA) e massa seca da raiz (MSR) de plantas de mandacaru (Cereus jamacaru D.C.) em função de diferentes soluções salinas e telados. Fortaleza-CE, 2015.

\begin{tabular}{cccllll}
\hline \multicolumn{7}{c}{ QUADRADO MÉDIO } \\
\hline FV & GL & AP & DC & MFT & MSPA & MSR \\
\hline Doses (D) & 4 & $0,62^{*}$ & $0,22^{\text {ns }}$ & $0,08^{* *}$ & $0,0002^{\text {ns }}$ & $0,0001^{\text {ns }}$ \\
Telados (T) & 2 & $9,84^{* *}$ & $2,69^{* *}$ & $0,66^{* *}$ & $0,0002^{* *}$ & $0,0002^{*}$ \\
(D x T) & 8 & $0,20^{\text {ns }}$ & $0,07^{\text {ns }}$ & $0,02^{*}$ & $0,0003^{*}$ & $0,0001^{\text {ns }}$ \\
Resíduo & 60 & 0,23 & 0,14 & 0,01 & 0,0001 & 0,00006 \\
\hline CV (\%) & 20,12 & 22,13 & 26,23 & 32,82 & 17,27 \\
Média & 2,41 & 1,67 & 0,41 & 0,04 & 0,05 \\
\hline FV=Fonte de variação; GL= Grau de liberdade; CV= Coeficiente de variação; Significativo a
\end{tabular}

$0,05\left(^{*}\right)$ e a $0,01\left(^{* *}\right)$ de probabilidade, respectivamente; ns- não significativo pelo teste $F$.

Com relação à sobrevivência das plântulas, o sombreamento de $30 \%$ com média de $33,64 \% \mu \mathrm{mol} \mathrm{m} \mathrm{m}^{-2} \mathrm{~s}^{-1}$ para os meses de instalação do trabalho, foi o que proporcionou menor sobrevivência das mudas de mandacaru. $O$ oposto pode-se afirmar para os sombreamentos de 50 e $70 \%$, no qual houve uma maior sobrevivência das plântulas de mandacaru para as condições de estudo (Tabela 2).

TABELA 2 - Médias de altura das plantas (AP), diâmetro do caule (DC), massa fresca total (MFT), massa seca da parte aérea (MSPA) e massa seca da raiz (MSR) de plântulas de mandacaru (Cereus jamacaru D.C.) submetidas a diferentes concentrações salinas e telados. Fortaleza-CE, 2015.

\begin{tabular}{|c|c|c|c|c|c|c|c|}
\hline Telado & Salinidade & AP & DC & MFT & MSPA & MSR & SOB \\
\hline 30 & $\begin{array}{l}0 \\
1 \\
2 \\
3 \\
4\end{array}$ & $\begin{array}{l}1,51 \mathrm{~b} \\
2,19 \mathrm{a} \\
1,65 \mathrm{~b} \\
1,61 \mathrm{~b} \\
1,50 \mathrm{~b}\end{array}$ & $\begin{array}{l}1,24 \mathrm{~b} \\
1,50 \mathrm{a} \\
1,32 \mathrm{~b} \\
1,18 \mathrm{~b} \\
1,34 \mathrm{~b}\end{array}$ & $\begin{array}{l}0,18 \mathrm{~b} \\
0,34 \mathrm{a} \\
0,23 \mathrm{~b} \\
0,17 \mathrm{~b} \\
0,18 \mathrm{~b}\end{array}$ & $\begin{array}{l}0,02 \mathrm{~b} \\
0,03 \mathrm{a} \\
0,03 \mathrm{a} \\
0,03 \mathrm{a} \\
0,02 \mathrm{~b}\end{array}$ & $\begin{array}{l}0,05 \mathrm{a} \\
0,04 \mathrm{~b} \\
0,05 \mathrm{a} \\
0,04 \mathrm{~b} \\
0,05 \mathrm{a}\end{array}$ & $\begin{array}{l}80 \% \\
90 \% \\
85 \% \\
85 \% \\
90 \%\end{array}$ \\
\hline 50 & $\begin{array}{l}0 \\
1 \\
2 \\
3 \\
4\end{array}$ & $\begin{array}{l}2,94 \mathrm{a} \\
3,11 \mathrm{a} \\
2,66 \mathrm{~b} \\
2,56 \mathrm{~b} \\
2,28 \mathrm{~b} \\
\end{array}$ & $\begin{array}{l}1,94 \mathrm{a} \\
1,99 \mathrm{a} \\
1,69 \mathrm{ab} \\
1,60 \mathrm{ab} \\
1,47 \mathrm{~b}\end{array}$ & $\begin{array}{l}0,67 \mathrm{a} \\
0,65 \mathrm{a} \\
0,45 \mathrm{~b} \\
0,44 \mathrm{~b} \\
0,37 \mathrm{~b}\end{array}$ & $\begin{array}{l}0,05 \mathrm{a} \\
0,06 \mathrm{a} \\
0,04 \mathrm{~b} \\
0,04 \mathrm{~b} \\
0,03 \mathrm{ab}\end{array}$ & $\begin{array}{l}0,04 \mathrm{~b} \\
0,04 \mathrm{ab} \\
0,05 \mathrm{a} \\
0,05 \mathrm{a} \\
0,05 \mathrm{a}\end{array}$ & $\begin{array}{r}100 \% \\
100 \% \\
100 \% \\
100 \% \\
90 \% \\
\end{array}$ \\
\hline 70 & $\begin{array}{l}0 \\
1 \\
2 \\
3 \\
4\end{array}$ & $\begin{array}{l}3,07 \mathrm{a} \\
2,79 \mathrm{ab} \\
2,85 \mathrm{a} \\
2,78 \mathrm{~b} \\
2,69 \mathrm{~b}\end{array}$ & $\begin{array}{l}2,05 \mathrm{a} \\
2,03 \mathrm{a} \\
1,97 \mathrm{a} \\
1,89 \mathrm{~b} \\
1,87 \mathrm{~b}\end{array}$ & $\begin{array}{l}0,22 \mathrm{c} \\
0,49 \mathrm{a} \\
0,47 \mathrm{ab} \\
0,43 \mathrm{~b} \\
0,45 \mathrm{~b}\end{array}$ & $\begin{array}{l}0,04 \mathrm{~b} \\
0,04 \mathrm{~b} \\
0,05 \mathrm{a} \\
0,04 \mathrm{~b} \\
0,04 \mathrm{~b}\end{array}$ & $\begin{array}{l}0,05 \mathrm{a} \\
0,05 \mathrm{a} \\
0,05 \mathrm{a} \\
0,05 \mathrm{a} \\
0,05 \mathrm{a}\end{array}$ & $\begin{array}{r}100 \% \\
95 \% \\
100 \% \\
100 \% \\
100 \% \\
\end{array}$ \\
\hline
\end{tabular}

Médias seguidas de letras iguais não diferem entre si, pelo teste de Tukey, a $5 \%$ de significância. Salinidade - $0\left(0 \mathrm{dS} \mathrm{m}^{-1}\right) ; 1$ (1-1,3 dS m $\left.\mathrm{m}^{-1}\right) ; 2\left(2-2,3 \mathrm{dS} \mathrm{m}^{-1}\right)$; $3\left(3-3,3 \mathrm{dS} \mathrm{m}^{-1}\right)$ e4 $\left(4-4,4 \mathrm{dS} \mathrm{m}^{-1}\right)$. 
Em termos gerais, observa-se que os melhores resultados foram obtidos para os sombreamentos de 50 e $70 \%$. No ambiente com sombreamento de $30 \%$, juntamente com as interações que 0 ambiente proporcionou, tais como alta temperatura, luminosidade e baixa umidade relativa do ar contribuíram para que as mudas cultivadas nesse tipo de ambiente apresentassem os piores valores médios observados para as variáveis analisadas (Tabela 2; Figura 1). Para todas as variáveis percebe-se que a partir do primeiro nível de salinidade testado ( $1-1,3$ dS $\mathrm{m}^{-1}$ ) as mudas de mandacaru foram afetadas pelo sal, principalmente as que permaneceram sob sombreamento de $30 \%$, pois tal ambiente proporcionou maior temperatura e luminosidade.

\section{Temperatura}

$-70 \%=50 \%=30 \%$

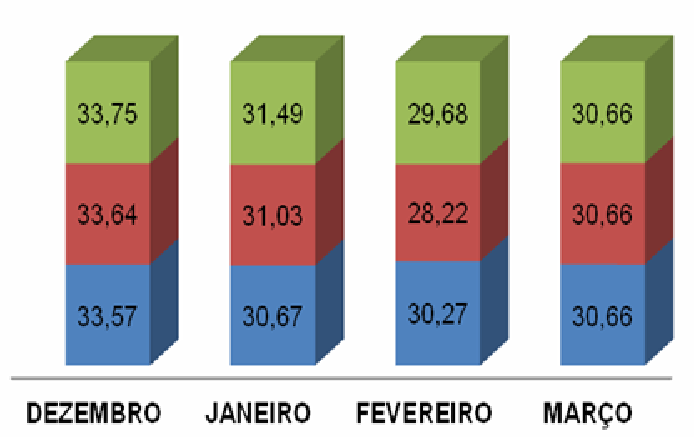

\section{Umidade Relativa (\%)}

$-70 \%=50 \%=30 \%$

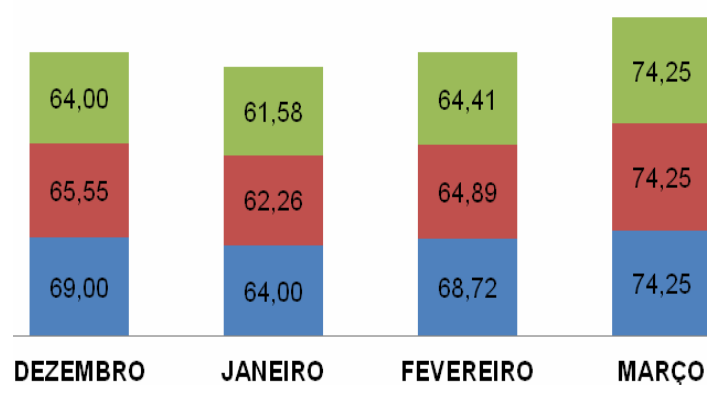

FIGURA 1 - Médias da temperatura e umidade relativa do ar durante dos meses de condução do trabalho (dezembro de 2014 - março 2015)

Tais resultados corroboram com os obtidos por GOMES et al., (2014), os quais trabalhando com tolerância e desenvolvimento de plântulas de Cereus jamacaru submetidas à salinidade observaram efeito negativo da salinidade sobre as variáveis analisadas.

De acordo com FERNANDES et al., (2003), o crescimento do vegetal é afetado pela salinidade devido ao estresse osmótico, toxicidade por excesso de íons, principalmente $\mathrm{Na}$ e $\mathrm{Cl}$, além do desequilíbrio nutricional. Estudos de SOUSA \& BEZERRA (2010) com desenvolvimento de mudas da suculenta Portulacaria afra Jacq. em água de irrigação salina também observaram redução na altura das plantas sob efeito da salinidade. Por outro lado, afirmam TAIZ \& ZEIGER (2013) que o maior crescimento de plantas em ambientes com menos luminosidade pode estar associado ao investimento em alongamento das células, podendo-se ter um aproveitamento mais eficiente da energia luminosa, assim como um maior controle da temperatura, favorecendo maior atividade fotossintética, comparado a plantas cultivadas sob radiação solar intensa.

A salinidade interferiu em todos os parâmetros analisados, podendo-se afirmar que essa espécie é sensível a baixas concentrações salinas sob condições de alta temperatura e umidade.

\section{CONCLUSÕES}

Com relação à sobrevivência das plântulas, o sombreamento de $30 \%$ (média de $33,64 \% \mu \mathrm{mol} \mathrm{m} \mathrm{m}^{-2} \mathrm{~s}^{-1}$ ) proporcionou menor sobrevivência das mudas de mandacaru. A maior sobrevivência das plântulas de mandacaru foi nos 
sombreamentos de 50 e $70 \%$. A partir do primeiro nível de salinidade testado (1 $1,3 \mathrm{dS} \mathrm{m}^{-1}$ ) as mudas de mandacaru foram afetadas pelo sal.

\section{AGRADECIMENTOS}

A Universidade Federal do Ceará, pelo apoio durante o desenvolvimento da pesquisa. A Empresa CASCAIS, que forneceu a matéria-prima para formação do substrato fibra de coco, utilizado na pesquisa para o Grupo de Pesquisas e Estudo em Floricultura CEFLOR.

\section{REFERÊNCIAS}

AASAMAA, K.; SÕBER, A. Stomatal sensitivities to changes in leaf water potential, air humidity, $\mathrm{CO}_{2}$ concentrationand light intensity, and the effect of abscisic acid on the sensitivities in six temperature deciduous tree species. Environmental and Experimental Botany, v. 71, p. $72-78,2011$.

CAVALCANTI, M. B. A.; RIBEIRO FILHO, N. M.; DIAS S. L.; BARBOSA, A. S.; DANTAS J. P. Avaliação nutricional do mandacaru com e sem espinhos. In: CONGRESSO NORTE-NORDESTE DE QUÍMICA, 2., 2008, João Pessoa: CEFETPB.

FERNANDES, A. R.; CARVALHO, J. G.; CURI, N.; GUIMARÃES, P. T.; PINTO, J. E. B. P. Crescimento de mudas de pupunheira (Bactris gasipaes H. B. K.) sob diferentes níveis de salinidade. Ciência Agrotécnica, Lavras, v. 2, n. 2 p. 278-284, Mar, 2003.

FERREIRA, D. F. SISVAR - Sistema de análise de variância. Versão 5.3. LavrasMG: UFLA, 2010.

GOMES, M. D. A.; GUIMARÃES, I. P.; MEDEIROS, M. A.; TORRES, S. B.; BENEDITO, C. P.; OLIVEIRA, F. T. R. Tolerância e desenvolvimento de plântulas de Cereus jamacaru P. submetidas à salinidade. II INOVAGRI International Meeting, 2014, Fortaleza, Brasil.

IBRAFLOR. Instituto Brasileiro de Floricultura. Mercado interno e Plano Safra 2013.1. Disponível em: <http://www.ibraflor.com/ns_mer_interno.php>. Acesso em: 04 set. 2015.

KÖPPEN, W. Climatologia: con un estudio de los climas de la tierra. México: Fondo de Cultura Econômica, 478p., 1948.

NOGUEIRA FILHO, F. P.; FREITAS, M. A. C.; PEREIRA, M. S.; BEZERRA, A. M. E.; LACERDA, C. F. Efeito de diferentes níveis de salinidade na água de irrigação e da luminosidade no crescimento de Plectranthus barbatus ANDR. In: INOVAGRI International Meeting \& WINOTEC - Workshop Internacional de Inovações Tecnológicas na Irrigação, 4., 2012, Fortaleza-CE. 
SANCHES, L. V. C; FERREIRA, M. J. C. L; BOSQUE, G. Teste de emergência e avaliação de desenvolvimento do cactos Melocactus deinacanthus em diversos tipos de substratos. Revista científica eletrônica de agronomia. Garça/SP, n.12, dez. 2007.

SEARLE, S. Y.; THOMAS, S.; GRIFFIN, K. L.; HORTON, T.; KORNFELD, A.; YAKIR, D.; HURRY, V.; TURNBULL, M. Leaf respiration and alternative oxidase in field-grown alpine grasses respond to natural changes in temperature and light. New Phytologist, v. 189, p. 1027 - 1039, 2011.

SOUSA, A. B. O.; BEZERRA, M. A. Desenvolvimento de mudas de Portulacaria afra. Jacq. oriundas de diferentes explantes e irrigadas com água salina. Simpósio Brasileiro de Salinidade, outubro de 2010, Fortaleza, CE, Brasil.

SOUZA, V. C; LORENZI, H. Botânica sistemática: guia ilustrado para identificação das famílias de angiospermas da flora brasileira, baseado em APG II. Nova Odessa: Instituto Plantarum, 2005.

TAIZ, L.; ZEIGER, E. Fisiologia vegetal. 5 ed. Porto Alegre: Artmed, 2013.

VIÉGAS, R.A.; SILVEIRA, J.A.G.; MELO, A.R.B.; LIMA JUNIOR, A.R.; QUEIROZ, J.E.; FAUSTO, M.J.M. Effects of $\mathrm{NaCl}$-salinity on growth and inorganic solute accumulation of young cashew plants, Revista Brasileira de Engenharia Agrícola e Ambiental, Campina Grande, v.5, n.2, p.216-222, 2001. 